\title{
The Causes and Consequences of COVID-19 Misperceptions: Understanding the Role of News and Social Media
}

\author{
Aengus Bridgman \\ McGill University \\ aengus.bridgman@mail.mcgill.ca \\ Peter John Loewen \\ University of Toronto \\ peter.loewen@utoronto.ca \\ Derek Ruths \\ McGill University \\ derek.ruths@mcgill.ca
}

Oleg Zhilin

McGill University

oleg.zhilin@mail.mcgill.ca

\author{
Eric Merkley \\ University of Toronto \\ eric.merkley@utoronto.ca \\ Taylor Owen \\ McGill University \\ taylor.owen@mcgill.ca \\ Lisa Teichmann \\ McGill University \\ lisa.teichmann@mail.mcgill.ca
}

May 1, 2020

\begin{abstract}
We investigate the relationship between media consumption, misinformation, and important attitudes and behaviours during the COVID-19 pandemic in Canada. We find that comparatively more misinformation circulates on social media platforms, while traditional news media tend to reinforce public health recommendations like social distancing. We find that exposure to social media is associated with misperceptions about COVID-19 while the inverse is true for news media. These misperceptions are in turn associated with lower compliance with social distancing measures. We thus draw a link from misinformation on social media to behaviours and attitudes that potentially magnify the scale and lethality of COVID-19.
\end{abstract}


The COVID-19 outbreak has been accompanied by a so-called "infodemic" - a global spread of misinformation that poses a serious problem for public health. Infodemics are concerning because the spread of false or misleading information has the capacity to change transmission patterns (Kim, Fast, and Markuzon 2019) and consequently the scale and lethality of an outbreak. This information can be shared by any media, but there is reason to be particularly concerned about the role that social media, such as Facebook and Twitter, play in incidentally boosting misperceptions. These platforms are increasingly relied upon as primary sources of news (Mitchell et al. 2016) and misinformation has been heavily documented on them (Vicario et al. 2016; Garrett 2019), with medical and health information being no-exception. Scholars have found misinformation related to vaccines (Radzikowski et al. 2016) and other virus outbreaks such as Ebola (Fung et al. 2016) and Zika to be prevalent on these platforms (Sharma et al. 2017).

During the COVID-19 outbreak, Twitter, Facebook and other platforms have engaged in efforts to combat misinformation but they have continued to receive widespread criticism that misinformation is still appearing on prominent pages and groups (NewsGuard 2020; Kouzy et al. 2020). Spending time in a media environment that contains misinformation is likely to change attitudes and behaviours. Even if users are not nested in networks that propagate misinformation, they are likely to be incidentally exposed to information from a variety of perspectives (Weeks et al. 2017; Fletcher and Nielsen 2018; Feezell 2018). Even a highly curated social media feed is thus likely to contain misinformation. As cumulative exposure to misinformation increases, users are likely to experience a reinforcement effect whereby familiarity leads to stronger belief (Dechêne et al. 2010).

While we have reason to be concerned, misinformation content typically makes up a low percentage of overall discussion of a topic (e.g. Fung et al. 2016) and mere exposure to misinformation does not guarantee belief in that misinformation. More research is needed to understand the extent and consequences of misinformation surrounding COVID-19 on social media. We thus ask: How prevalent is misinformation surrounding COVID-19 on social media, and how does this compare to news media? Does the type of media one is exposed to influence social distancing behaviours and beliefs about COVID-19? And Is there a link between COVID-19 misinformation and perceptions of the pandemic's severity and compliance with social distancing recommendations?

\section{Design and Method}

To answer these questions, we draw on three data sets and a sequential mixed method approach to better understand the consequences of online misinformation for important behaviours and attitudes. First, we collected nearly 2.5 million tweets explicitly referring to COVID-19 in the Canadian context. Second, we collected just over 9 thousand articles nom nineteen Canadian English-language news sites from the same time period. We coded both of these media sets for misinformation and public health recommendations. Third, we conducted a nationally representative survey that included questions related to media consumption habits, COVID-19 perceptions and misperceptions, and social distancing compliance.

Canadian social media and news data were collected from March $26^{\text {th }}$ to April $6^{\text {th }}$. We collected all English-language tweets from a set of 620,000 users that have been determined to be likely

Canadians. For inclusion, a given user must self-identify as Canadian-based, follow a large number of Canadian political elite accounts, or frequently use Canadian-specific hashtags. News media was 
collected from nineteen prominent Canadian news sites with active RSS feeds. These tweets and news articles were searched for "covid" or "coronavirus", leaving a sample of 2.25 million tweets and 9,148 news articles.

Of the COVID-19 related content, we searched for terms associated with four categories of misinformation that circulated in the early days of the COVID-19 outbreak: that COVID-19 was no more serious than the flu, that vitamin $\mathrm{C}$ or other supplements will prevent contraction of the virus, that the initial animal-to-human transfer of the virus was the direct result of eating bats, or that COVID-19 was a hoax or conspiracy. Given that content mentioning these terms could be in a variety of contexts, we manually reviewed a random sample of 500 tweets from each of the three pieces of misinformation. Each tweet was coded into four categories: propagating misinformation, or clarifying that the misinformation as misinformation, unrelated, or referring to the misinformation without comment.

The overall level of misinformation on Twitter was inferred from the percentage of the handcoded content that included misinformation. Each news article that included relevant keywords was similarly coded. The volume of the news mentioning these terms was sufficiently low that random sampling was not required. To identify recommendations for safe practices during a pandemic, we used a similar keyword search for terms associated with particular recommendations: 1) social distancing including staying at home, staying at least 6 feet or 2 meters away and avoiding gatherings; and 2) washing hands and not touching any part of your face. ${ }^{1}$

For survey data, we used a sample of nearly 2,500 Canadian citizens 18 years or older drawn from a probability-based online national panel fielded from April 2-6, 2020. Quotas we set on age, gender, region, and language to ensure sample representativeness, and data was further weighted within region by gender and age based on the 2016 Canadian census.

We measure levels of COVID-19 misperceptions by asking respondents to rate the truthfulness of a series of nine false claims, such as the coronavirus being no worse than the seasonal flu or that it can be warded off with Vitamin C. Each was asked on a scale from definitely false (0) to definitely true (5). Principal Components Analysis (PCA) reveal these questions load on one dimension. The items have a Cronbach's Alpha of 0.88 . We thus construct a $0-1$ scale of misperceptions from these items.

We evaluate COVID-19 risk perceptions with a trio of questions asking respondents their level of concern about the coronavirus pandemic, and how serious of a threat they believe the pandemic to be for themselves and for Canadians, respectively. Each were asked on a scale from not at all (0) to very (4). We construct a continuous index with these items.

We quantify social distancing by asking respondents to indicate which of a series of behaviours they had undertaken in response to the pandemic, such as working from home or avoiding inperson contact with friends, family, and acquaintances. We use PCA to identify 2 distinct dimensions in our questions. One dimension includes factors strongly determined by occupation, such as working from home and switching to online meetings. The other dimension contains more

\footnotetext{
${ }^{1}$ Further details on the media collection strategy and hand-coding schema are available in the supporting materials.
} 
inclusive behaviours such as avoiding contact, travel, and crowded places. We use this latter dimension in our analyses as predicted by the PCA. The factor loadings can be found in Table A1 of the supporting materials.

We gauge news consumption and social media consumption by asking respondents to identify news outlets and social media applications they have used over the past week for political news. The list of news outlets included 17 organizations such as mainstream sources like CBC and Global, and partisan outlets like Rebel Media and National Observer. The list of social media applications included 10 options including Facebook, Twitter, YouTube, and Instagram. We sum the number of outlets of applications for each and take the log to adjust for extreme values. We measure offline political discussion with an index based on questions asking how often respondents have discussed politics with family, friends, and acquaintances over the past week. Descriptions of our primary variables can be found in Table A2 of the supporting materials.

We evaluate our hypotheses using a standard design that evaluates the association between our explanatory and outcome variables controlling for other observable factors we measured. In practice, randomly assigning social media exposure is impractical, while randomly assigning misinformation is unethical. This approach allows us to describe these relationships, though we cannot make definite claims to causality.

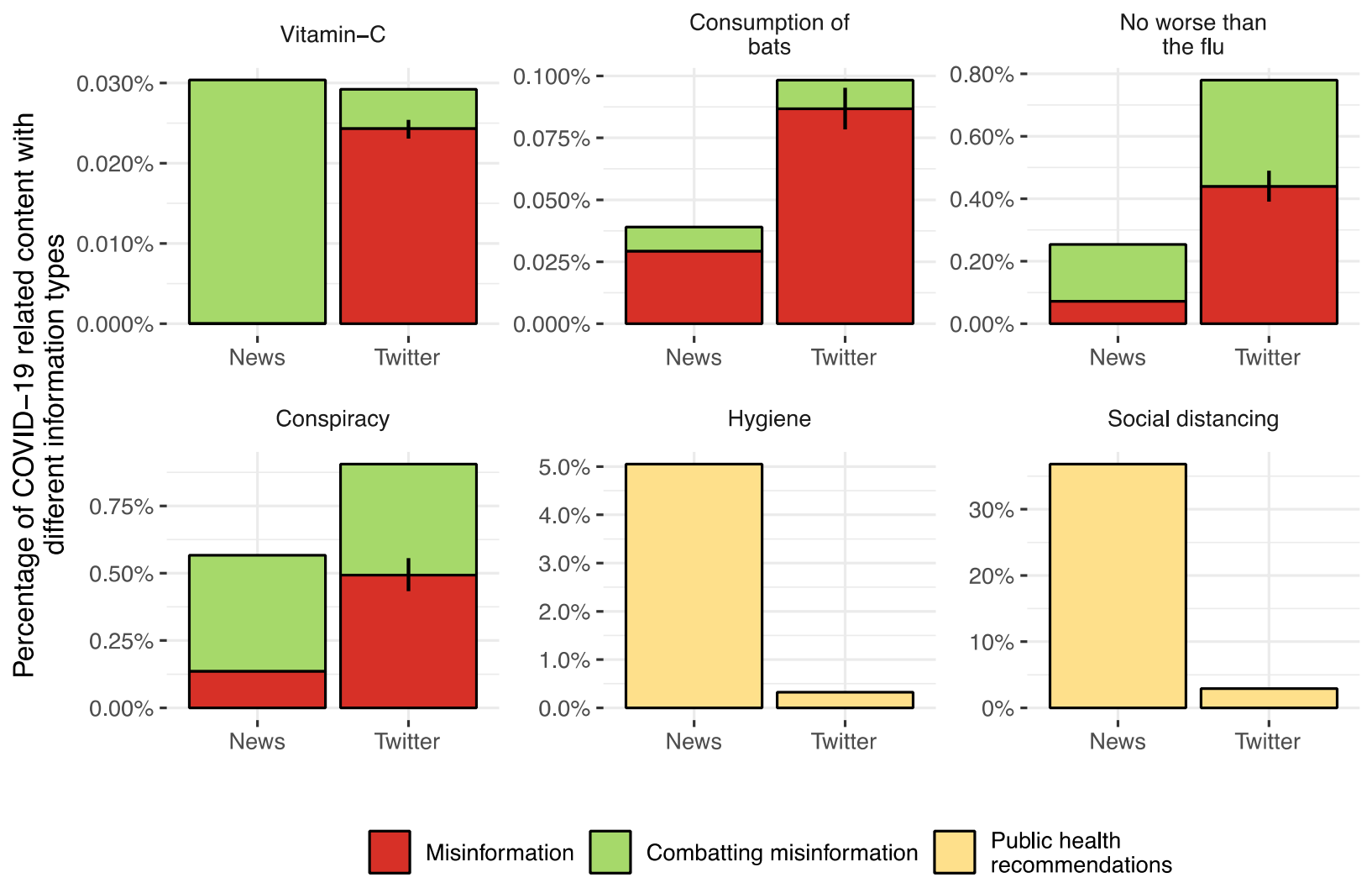

Figure 1. Relative frequencies of COVID-19 related information. Figure 1 plots the percentage of all COVID-19 related content that includes reference to a particular theme. Confidence intervals are shown only for the tweets where a random sample was manually categorized and the results extrapolated to all tweets in the corpus. 


\section{Findings}

Finding 1: Misinformation about COVID-19 is circulated more on social media as compared to traditional media.

We first compare the presence of misinformation on social and news media during the initial months of the outbreak and find, consistent with the other country cases, that the social media environment has high levels of misinformation in Canada (Vicario et al. 2016; Chadwick and Vaccari 2019). We also found that recommendations for safe practices during the pandemic (e.g. washing hands, social distancing) appeared much more frequently in the Canadian news media. These findings are consistent with literature examining fake news which finds a large difference in information quality across media (Al-Rawi 2019; Guess and Nyhan 2018).

We find large differences between the quality of information shared about COVID-19 on traditional news and social media. Figure 1 shows the percentage of COVID-19 related content that contains information linked to a particular theme. The plot reports the prevalence of information on both social and news media for: 1) three specific pieces of misinformation; 2) a general set of content that describes the pandemic itself as a conspiracy or a hoax; and 3) advice about hygiene and social distancing during the pandemic. We differentiate content that shared misinformation (red in the plot) from content that debunked misinformation (green in the plot).

There are large differences between the levels of misinformation on social media and news media. Misinformation was comparatively more common on social media across all measures, while combatting misinformation was relatively more common in traditional news. Meanwhile, advice on hygiene and social distancing appeared much more frequently in news media. Note that higher percentages are to be expected for longer format news articles since we rely on keyword searches for identification. This makes the misinformation findings even starker - despite much higher average word counts, far fewer news articles propagate misinformation.

Finding 2: There is a strong association between social media exposure and misperceptions about COVID-19. The inverse is true for exposure to traditional news.

Among our survey respondents we find a corresponding strong association between social media exposure and misperceptions about COVID-19. These results are plotted in Figure 2, with controls included for both socioeconomic characteristics and demographics. Moving from no social media exposure to its maximum is expected to increase one's misperceptions of COVID-19 by 0.22 on the 0-1 scale and decreased self-reported social distancing compliance by 0.12 on that same scale.

This result stands in stark contrast with the observed relationship between traditional news exposure and our outcome measures. Traditional news exposure is positively associated with correct perceptions regarding COVID-19. Moving from no news exposure to its highest level is expected to reduce misperceptions by 0.12 on the $0-1$ scale and to increase social distancing compliance by 0.28 on that same scale. The effects are similarly plotted in Figure 2. Social media usage appears to be correlated with COVID-19 misperceptions, suggesting these misperceptions are partially a result of misinformation on social media. The same cannot be said of traditional news exposure. These findings are consistent with other work that links exposure to misinformation to misperceptions (Jamieson and Albarracín 2020; Garrett, Weeks, and Neo 2016). 


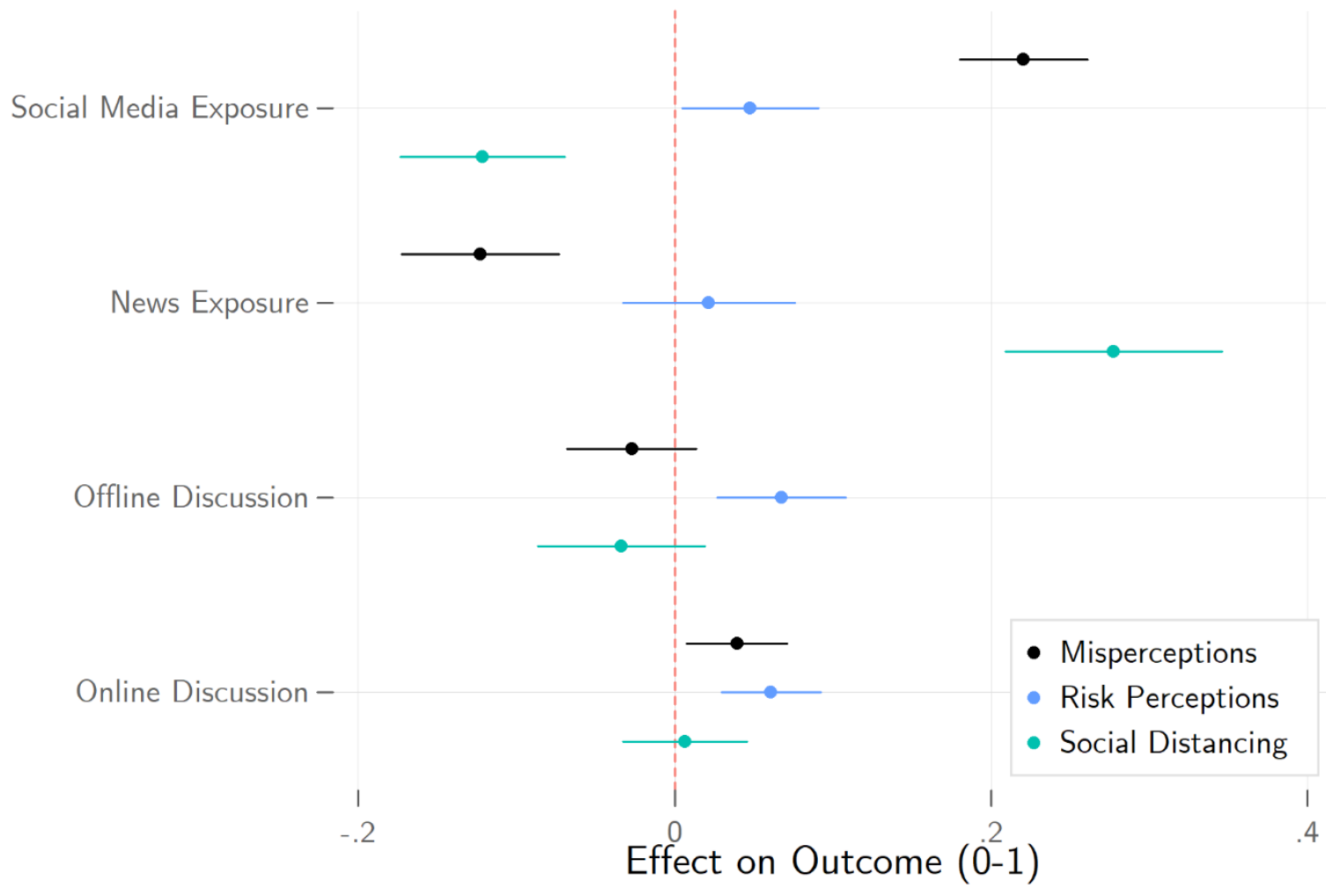

Figure 2. Effects of Media and Discussion on COVID-19 Misperceptions, Risk Perceptions, and Social Distancing Practice. Figure 2 plots the coefficients from three Ordinary Least Squares regression models. All measures scaled from 0-1, so effects can be interpreted as going from the minimum to the maximum of explanatory variables on the $0-1$ scales of each outcome. Additional controls for income, education, age, gender, religiosity, language, and region not plotted. 95\% confidence intervals plotted.

Finding 3: Misperceptions about the pandemic are associated with lower levels of risk perceptions and social distancing compliance.

COVID-19 misperceptions are also powerfully associated with lower levels of social distancing compliance. Moving from the lowest level of COVID-19 misperceptions to its maximum is associated with a reduction of one's social distancing by 0.39 on the $0-1$ scale. The previously observed relationship between social media exposure and misperceptions disappears, suggestive of a mediated relationship. That is, social media exposure increases misperceptions, which then reduces social distancing compliance. Misinformation is also weakly associated with lower COVID-19 risk perceptions, but this is only marginally significant ( $\mathrm{p} \sim 0.08$ ). Estimates from our models using COVID-19 concern as the outcome can be found in the left panel of Figure 3, while social distancing can be found in the right panel.

Finally, we also see that the relationship between misinformation and both social distancing compliance and COVID-19 concern hold when including controls for science literacy and a number of fundamental predispositions that are likely associated with both misperceptions and following the 
advice of scientific experts, such as anti-intellectualism, pseudoscientific beliefs, and left-right ideology. These estimates can similarly be found in Figure 3.

Misperceptions are most meaningful when they impact behaviours in dangerous ways. During a pandemic, misperceptions can be fatal. In this case, we find that misperceptions are associated with reduced COVID-19 risk perceptions and with lower compliance with social distancing measures. We continue to find strong effects after controlling for socio-economic characteristics as well as scientific literacy. After accounting for the effect of misperceptions on social distancing noncompliance, social media usage no longer has a significant association with non-compliance, providing some suggestive evidence that social media may lead to less social distancing compliance through its effect on COVID-19 misperceptions.
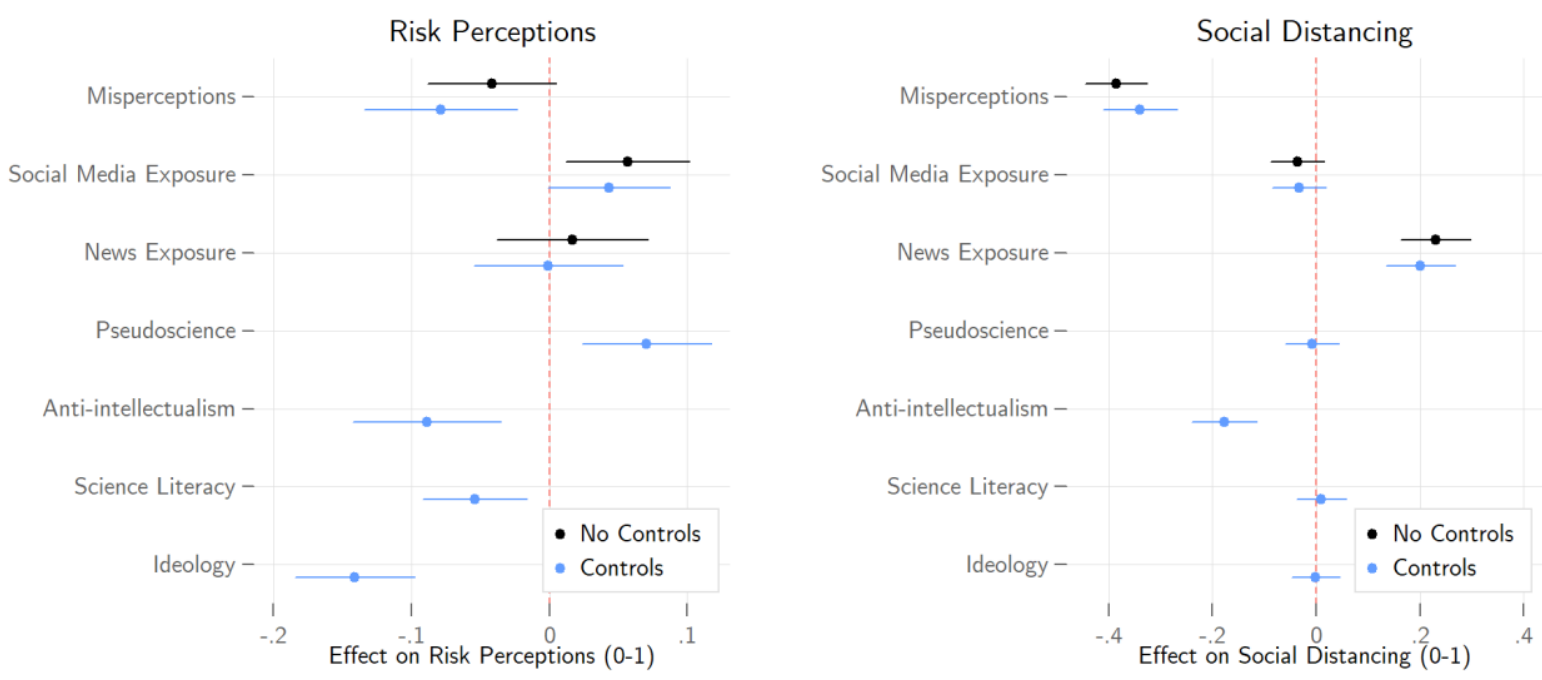

Figure 3. Effects of COVID-19 Misperceptions on Risk Perceptions (Left) and Social Distancing (Right). Figure 3 plots the coefficients from Ordinary Least Squares regression models. Left panel plots the effects of misperceptions on COVID-19 risk perceptions with and without science literacy and predispositions as controls. Right panel does the same for social distancing. All measures scaled from 0-1, so effects can be interpreted as going from the minimum to the maximum of explanatory variables on the $0-1$ scales of each outcome. Additional controls for online and offline discussion, income, education, age, gender, religiosity, language, and region not plotted. 95\% confidence intervals plotted.

\section{Discussion}

Highly polarized political environments and media ecosystems can lead to the spread of misinformation, such as in the United States during the COVID-19 outbreak (Allcott et al. 2020; Motta, Stecula, and Farhart 2020). But even in healthy media ecosystems with less partisan news (Owen et al. 2020), social media can continue to facilitate the spread of misinformation. While some social media companies have made efforts to suppress misinformation on their platforms, there continues to be a high level of misinformation relative to news media. We have shown a higher prevalence of misinformation regarding COVID-19 on social media and shown that those who self- 
report higher exposure to social media have greater misperceptions about the pandemic.

Misperceptions are most meaningful when they impact behaviours in dangerous ways; and during a pandemic, misperceptions can be fatal.

We have further shown that these misperceptions are associated with reduced COVID-19 risk perceptions and lower compliance with social distancing measures. There is a real danger that without concerted efforts to reduce the amount of misinformation shared on social media, the largescale social efforts required to combat COVID-19 will be undermined. We argue that the COVID19 epidemic clearly illustrates how use of social media can contribute to misperceptions and consequently encourage or promote behaviours that put individuals and societies at risk. We join others in calling for social media companies to put greater focus on flattening the curve of misinformation (Donovan 2020).

\section{References}

Allcott, Hunt, Levi Boxell, Jacob C Conway, Matthew Gentzkow, Michael Thaler, and David Y Yang. 2020. "Polarization and Public Health: Partisan Differences in Social Distancing during the Coronavirus Pandemic.” Working Paper 26946. Working Paper Series. National Bureau of Economic Research. https://doi.org/10.3386/w26946.

Al-Rawi, Ahmed. 2019. "Gatekeeping Fake News Discourses on Mainstream Media Versus Social Media." Social Science Computer Review 37 (6): 687-704. https://doi.org/10.1177/0894439318795849.

Chadwick, Andrew, and Cristian Vaccari. 2019. "News Sharing on UK Social Media: Misinformation, Disinformation, and Correction." Report. Loughborough University. https://repository.lboro.ac.uk/articles/News_sharing_on_UK_social_media_misinformation_disinf ormation_and_correction/9471269.

Dechêne, Alice, Christoph Stahl, Jochim Hansen, and Michaela Wänke. 2010. "The Truth About the Truth: A Meta-Analytic Review of the Truth Effect.” Personality and Social Psychology Review 14 (2): 238-57. https://doi.org/10.1177/1088868309352251.

Donovan, Joan. 2020. "Social-Media Companies Must Flatten the Curve of Misinformation." Nature, April. https://doi.org/10.1038/d41586-020-01107-z.

Feezell, Jessica T. 2018. “Agenda Setting through Social Media: The Importance of Incidental News Exposure and Social Filtering in the Digital Era." Political Research Quarterly 71 (2): 482-94. https://doi.org/10.1177/1065912917744895.

Fletcher, Richard, and Rasmus Kleis Nielsen. 2018. “Are People Incidentally Exposed to News on Social Media? A Comparative Analysis." New Media \& Society 20 (7): 2450-68. https://doi.org/10.1177/1461444817724170.

Fung, Isaac Chun-Hai, King-Wa Fu, Chung-Hong Chan, Benedict Shing Bun Chan, Chi-Ngai Cheung, Thomas Abraham, and Zion Tsz Ho Tse. 2016. "Social Media's Initial Reaction to Information and Misinformation on Ebola, August 2014: Facts and Rumors.” Public Health Reports 131 (3): 461-73. https://doi.org/10.1177/003335491613100312.

Garrett, R. Kelly. 2019. "Social Media's Contribution to Political Misperceptions in U.S. Presidential Elections.” PLoS ONE 14 (3). https://doi.org/10.1371/journal.pone.0213500. 
Garrett, R. Kelly, Brian E. Weeks, and Rachel L. Neo. 2016. "Driving a Wedge Between Evidence and Beliefs: How Online Ideological News Exposure Promotes Political Misperceptions." Journal of Computer-Mediated Communication 21 (5): 331-48. https://doi.org/10.1111/jcc4.12164.

Guess, Andrew, and Brendan Nyhan. 2018. "Selective Exposure to Misinformation: Evidence from the Consumption of Fake News during the 2016 U.S. Presidential Campaign." European Research Council, 49 .

Jamieson, Kathleen Hall, and Dolores Albarracín. 2020. "The Relation between Media Consumption and Misinformation at the Outset of the SARS-CoV-2 Pandemic in the US." Harvard Kennedy School Misinformation Review, no. 2 (April). https://doi.org/10.37016/mr-2020-012.

Kim, Louis, Shannon M. Fast, and Natasha Markuzon. 2019. "Incorporating Media Data into a Model of Infectious Disease Transmission.” Edited by Jeffrey Shaman. PLOS ONE 14 (2): e0197646. https://doi.org/10.1371/journal.pone.0197646.

Kouzy, Ramez, Joseph Abi Jaoude, Afif Kraitem, Molly B El Alam, Basil Karam, Elio Adib, Jabra Zarka, Cindy Traboulsi, Elie W Akl, and Khalil Baddour. 2020. "Coronavirus Goes Viral: Quantifying the COVID-19 Misinformation Epidemic on Twitter.” Cureus 12 (3). https://doi.org/10.7759/cureus.7255.

Mitchell, Amy, Jeffrey Gottfried, Michael Barthel, and Elisa Shearer. 2016. "The Modern News Consumer." Pew Research Center's Journalism Project (blog). July 7, 2016. https://www.journalism.org/2016/07/07/the-modern-news-consumer/.

Motta, Matthew, Dominik Stecula, and Christina E. Farhart. 2020. "How Right-Leaning Media Coverage of COVID-19 Facilitated the Spread of Misinformation in the Early Stages of the Pandemic." Preprint. SocArXiv. https://doi.org/10.31235/osf.io/a8r3p.

NewsGuard. 2020. “Superspreaders.” https://www.newsguardtech.com/superspreaders/.

Owen, Taylor, Aengus Bridgman, Robert Gorwa, Peter Loewen, Stephanie MacLellan, Eric Merkley, Derek Ruths, and Oleg Zhilin. 2020. "Lessons in Resilience." Public Policy Forum.

Radzikowski, Jacek, Anthony Stefanidis, Kathryn H. Jacobsen, Arie Croitoru, Andrew Crooks, and Paul L. Delamater. 2016. “The Measles Vaccination Narrative in Twitter: A Quantitative Analysis.” JMIR Public Health and Surveillance 2 (1): e1. https://doi.org/10.2196/publichealth.5059.

Sharma, Megha, Kapil Yadav, Nitika Yadav, and Keith C. Ferdinand. 2017. "Zika Virus Pandemic-Analysis of Facebook as a Social Media Health Information Platform." American Journal of Infection Control 45 (3): 301-2. https://doi.org/10.1016/j.ajic.2016.08.022.

Vicario, Michela Del, Alessandro Bessi, Fabiana Zollo, Fabio Petroni, Antonio Scala, Guido Caldarelli, H. Eugene Stanley, and Walter Quattrociocchi. 2016. "The Spreading of Misinformation Online." Proceedings of the National Academy of Sciences 113 (3): 554-59. https://doi.org/10.1073/pnas.1517441113.

Weeks, Brian E., Daniel S. Lane, Dam Hee Kim, Slgi S. Lee, and Nojin Kwak. 2017. "Incidental Exposure, Selective Exposure, and Political Information Sharing: Integrating Online Exposure Patterns and Expression on Social Media." Journal of Computer-Mediated Communication 22 (6): 363-79. https://doi.org/10.1111/jcc4.12199. 


\section{Supporting Materials}

\section{Twitter Collection Strategy}

We drew upon a large-scale Twitter data collection effort in the Canadian context. 620, 000 Twitter users were identified as relevant to the Canadian conversation on the platform. For inclusion, a user needed to: a) have been manually identified as a politician, journalist, academic, or public personality $(\mathrm{N}=6000)$; b) identify themselves as Canadian or as located in Canada in their Twitter profile; c) have recently tweeted with Canadian-specific hashtags (e.g. \#toronto) AND have followed at least 10 Canadian politicians and news personalities; d) be in the top percentile for following Canadian politicians and news personalities. All tweets for this population have been collected since January 1 , 2020, with the Tweets used in this study running from 1 week before the survey was in the field to the last day the survey was in the field.

\section{Media Collection Strategy}

The html of Canadian news sites with active RSS feeds was collected then processed using newspaper3k in Python. Data was collected for the following English-language publications: Global News, CBC, Toronto Star, Financial Post, National Post, Winnipeg Free Press, Toronto Sun, Montreal Gazette, Huffington Post, Ottawa Citizen, Calgary Herald, Edmonton Journal, National Observer, Macleans, iPolitics, Winnipeg Sun, Vancouver Sun, Vancouver Prince, and Edmonton Sun. All articles posted between 1 week before the survey was put into the field and the last day the survey was in the field were included.

\section{$\underline{\text { Coding Schema }}$}

We used the following dictionary schema to identify COVID-19 related content. The hygiene and social distancing language was taken from official health recommendations from:

https://www.canada.ca/en/public-health/services/diseases/2019-novel-coronavirusinfection/guidance-documents.html (accessed April 20, 2020). Each document was tokenized and then token-matching was effected for the following terms.

COVID-19:('coronavirus','covid','covid-19')

Flu: ('flu')

Bat: ('bat','bats')

Vitamin-C: ('vitamin-c','vitamin c'),

Hygiene: ('hand wash', 'wash hands', 'hand washing', '30 seconds', 'thirty seconds', 'with soap', 'wash your hands', 'hand sanitizer', 'avoid touching your eyes', 'avoid touching your face', 'avoid touching your mouth')

Social distancing: ('work from home', 'stay home', 'stay at home', 'avoid all non-essential trips', 'gather in groups', 'avoid places', 'avoid public', 'avoid crowds', 'avoid gatherings', 'grocery delivery', 'avoid large gatherings', 'avoid small gatherings', 'and small gatherings', 'and large gatherings', 'limit 
events', 'limit meetings', 'self-isolate', 'isolation', 'must isolate', 'social distance', 'social distancing', 'six feet', ' 6 ft', '2 meter', '2 meters', 'maintain distance'

Conspiracy: ('hoax', 'fraud', 'deception', 'swindle', 'dupe', 'con', 'trick', 'deceive', 'scam', 'scheme', 'racket', 'overblown', 'exaggerated', 'overdone', 'inflated', 'embellished', 'hyperbolic', 'conspiracy', 'hyperbole', 'harmless')

Supporting Tables for Individual-level Analysis

Table A1 shows factor loadings for social distancing measures. Factor 1 generally captures compliance with physical distancing recommendations and is used throughout the paper. Factor 2 contains behaviours strongly determined by occupation, such as working from home and switching to online meetings. Table A2 provides the descriptions for the explanatory and outcome variables.

Table A1. Factor loadings for social distancing measures

\begin{tabular}{lccc}
\hline Variable & Factor 1 & Factor 2 & Uniqueness \\
\hline Worked from home & -0.164 & 0.733 & 0.437 \\
Avoided bars, restaurants, and crowds & 0.767 & -0.001 & 0.412 \\
Avoided grocery stores at peak times & 0.601 & 0.210 & 0.595 \\
Avoided in-person contact & 0.707 & 0.068 & 0.495 \\
Stocked up on provisions & 0.258 & 0.404 & 0.770 \\
Kept distance of two metres & 0.767 & -0.001 & 0.412 \\
Switched to virtual meetings & 0.266 & 0.662 & 0.492 \\
Switched to online shopping & 0.238 & 0.614 & 0.566 \\
Avoided domestic travel & 0.715 & 0.158 & 0.465 \\
Avoided public transit & 0.705 & 0.187 & 0.468 \\
\hline
\end{tabular}


Table A2. Variable Descriptions

\begin{tabular}{llcc}
\hline Measure & Description & Mean & SD \\
\hline & $\begin{array}{l}\text { 0-1;1) How serious of a threat do you think the coronavirus is } \\
\text { to yourself? (very, somewhat, not very, not at all); 2) How } \\
\text { serious of a threat do you think the coronavirus is to } \\
\text { Canadians? (very, somewhat, not very, not at all); 3) How } \\
\text { concerned are you about the coronavirus pandemic? (very, } \\
\text { Risk Perceptions }\end{array}$ & 0.78 & 0.21 \\
& 0-1; First dimension of PCA. More details in Table A1 & 0.75 & 0.26 \\
\hline Social Distancing & & & \\
\hline
\end{tabular}

0-1; Please rate the truthfulness of the following claims: 1) The coronavirus is no worse than the seasonal flu; 2) Drinking water every 15 minutes will help prevent the coronavirus; 3) The Chinese government developed the coronavirus as a bioweapon; 4) Homeopathy and home remedies can help manage and prevent the coronavirus; 5) The coronavirus was Misperceptions caused by the consumption of bats in China; 6) The coronavirus will go away by the summer; 7) Vitamin C can ward off the coronavirus; 8) There is a vaccine for the coronavirus that national governments and pharmaceutical companies won't release; 9) High temperatures, such as from saunas and hair dryers, can kill the coronavirus (definitely true, probably true, probably false, definitely false, unsure)

Logged, 0-1; Over the past week, which of the follow social media applications did you use to watch, read, or listen to news Social Media Exposure about politics? Please select all that apply. 1) Twitter 2) Facebook; 3) Instagram; 4) Youtube; 5) Reddit; 6) LinkedIn; 7) Tumblr; 8) WhatsApp; 9) Snapchat; 10) WeChat; 11) Another application

0-1; Over the past week, which of the follow news media outlets did you watch, read, or listen to for news about politics? Please select all that apply. 1) CBC; 2) CTV; 3) Global; 4) CityNews; 5) Globe and Mail; 6) National Post; 7) Toronto Star; 8) Local newspaper; 9) TVA (QC only); 10) TV5 (QC News Exposure $\quad$ only); 11) La Presse (QC only); 12) Journal de Montreal (QC only); 13) Journal de Quebec (QC only); 14) Le Devoir (QC $0.42 \quad 0.19$ only); 15) Radio-Canada (QC only); 16) Rebel Media; 17) National Observer; 18) Toronto Sun; 19) The Tyee; 20) Post Millennial; 21) APTN; 22) True North News; 23) Press Progress; 24) Huffington Post; 25) Another outlet

0-1; How often in the past week did you talk about politics or

Offline Discussion public affairs with the following people? 1) Your family; 2) Your friends; 3) Your co-workers (never, once, a few times, $0.40 \quad 0.26$ almost every day, daily, don't know) 
0-1; How many days in the past week did you talk about politics

Online Discussion or public affairs with anyone via the Internet, including social media, e-mail, chat rooms, and micro-blogging sites? (never, once, a few times, almost every day, daily, don't know)

Pseudoscience Beliefs

$0-1$; 1) Some numbers are especially lucky for people; 2) Faith healing is a valid alternative to conventional medicine; 3 )

Homeopathy is a valid alternative to conventional medicine; 4) Some ancient civilizations were visited by extraterrestrials; 5)

Some people possess psychic powers; 6 ) The position of the planets have an influence on the events of everyday life (strongly, somewhat, neither agree/disagree)

$0-1$; Below is a list of groups and institutions in society. Please tell us the degree to which you trust or distrust members of

Anti-intellectualism these groups or institutions: 1) Experts; 2) Economists; 3) Scientists; 4) Doctors and medical professionals; 5) University professors (distrust a lot, distrust somewhat, neither, trust somewhat, trust a lot, don't know)

$0-1 ; 1)$ The center of the Earth is very hot; 2) The continents have been moving their location for millions of years and will continue to move; 3) All radio-activity is man-made; 4)

Science Literacy Electrons are smaller than atoms; 5) Lasers work by focusing sound waves; 6) It is the father's gene that decides whether the baby is a boy or a girl; 7) Antibiotics kill viruses as well as bacteria.

$0-1 ; 1)$ The government should take measures to reduce differences in income levels; 2) Protecting the environment is more important than creating jobs; 3) Canada should increase the number of immigrants it admits each year; 4) People who Ideology don't get ahead should blame themselves, not the system; 5) The government should see to it that everyone has a decent standard of living (Strongly, somewhat, neither agree/disagree). Each item coded in left-wing (-1) and right-wing (1) direction. Don't knows and neither coded as neutral (0) 
The two following regression tables provide the full coefficient list for Figures 2 and 3 in the main body of the paper.

Table A3. OLS Estimates for Figure 2

\begin{tabular}{lccc}
\hline & Misperceptions & Risk & Compliance \\
& 1 & 2 & 3 \\
\hline Social Media & $0.22^{* * *}$ & $0.05^{* *}$ & $-0.12^{* * *}$ \\
News Exposure & $(0.02)$ & $(0.02)$ & $(0.03)$ \\
& $-0.12^{* * *}$ & 0.02 & $0.28^{* * *}$ \\
Offline Discussion & $(0.03)$ & $(0.03)$ & $(0.03)$ \\
& -0.03 & $0.07 * * *$ & -0.03 \\
Online Discussion & $(0.02)$ & $(0.02)$ & $(0.03)$ \\
& $0.04^{* *}$ & $0.06^{* * *}$ & 0.01 \\
Income & $(0.02)$ & $(0.02)$ & $(0.02)$ \\
& $0.01^{* * *}$ & 0.00 & $-0.01^{* * *}$ \\
Education & $(0.00)$ & $(0.00)$ & $(0.00)$ \\
Age & $-0.02^{* * *}$ & $-0.00^{*}$ & $0.02^{* * *}$ \\
& $(0.00)$ & $(0.00)$ & $(0.00)$ \\
Religiosity & $-0.00^{* * *}$ & $0.00^{* * *}$ & $0.00^{* * *}$ \\
Urban Density & $(0.00)$ & $(0.00)$ & $(0.00)$ \\
French & $0.06^{* * *}$ & $0.02^{* * *}$ & $-0.02^{* * *}$ \\
& $(0.00)$ & $(0.00)$ & $(0.00)$ \\
Female & 0.00 & 0.00 & $-0.01^{* * *}$ \\
Constant & $(0.00)$ & $(0.00)$ & $(0.00)$ \\
\hline R 2 & 0.02 & $-0.08^{* * *}$ & $-0.10^{* * *}$ \\
N & $(0.02)$ & $(0.03)$ & $(0.03)$ \\
\hline Note: Stan & $-0.04^{* * *}$ & 0.00 & $0.10^{* * *}$ \\
& $(0.01)$ & $(0.01)$ & $(0.01)$ \\
& $0.22^{* * *}$ & $0.05^{* *}$ & $-0.12^{* * *}$ \\
\hline
\end{tabular}

Note: Standard errors in parentheses; ${ }^{*} \mathrm{p}<0.1,{ }^{* *} \mathrm{p}<0.05,{ }^{* * *} \mathrm{p}<0.01$. Controls for region (Atlantic, Quebec, Ontario, West) 
Table A4. OLS Estimates for Figure 3

\begin{tabular}{|c|c|c|c|c|}
\hline & $\begin{array}{c}\text { Risk } \\
1\end{array}$ & $\begin{array}{c}\text { Social } \\
\text { Distancing } \\
2\end{array}$ & $\begin{array}{c}\text { Risk } \\
3\end{array}$ & $\begin{array}{c}\text { Social } \\
\text { Distancing } \\
4\end{array}$ \\
\hline Misperceptions & $\begin{array}{l}-0.04^{*} \\
(0.02)\end{array}$ & $\begin{array}{c}-0.39 * * * \\
(0.03)\end{array}$ & $\begin{array}{c}-0.08^{* * *} * \\
(0.03)\end{array}$ & $\begin{array}{c}-0.34 * * * \\
(0.04)\end{array}$ \\
\hline Social Media & $\begin{array}{l}0.06^{* *} \\
(0.02)\end{array}$ & $\begin{array}{l}-0.04 \\
(0.03)\end{array}$ & $\begin{array}{l}0.04^{*} \\
(0.02)\end{array}$ & $\begin{array}{l}-0.03 \\
(0.03)\end{array}$ \\
\hline News Exposure & $\begin{array}{c}0.02 \\
(0.03)\end{array}$ & $\begin{array}{c}0.23^{* * *} \\
(0.03)\end{array}$ & $\begin{array}{l}-0.00 \\
(0.03)\end{array}$ & $\begin{array}{c}0.20^{* * *} \\
(0.03)\end{array}$ \\
\hline Offline Discussion & $\begin{array}{c}0.07 * * * \\
(0.02)\end{array}$ & $\begin{array}{c}-0.04^{*} \\
(0.03)\end{array}$ & $\begin{array}{c}0.06^{* * *} \\
(0.02)\end{array}$ & $\begin{array}{c}-0.06^{* *} \\
(0.03)\end{array}$ \\
\hline Online Discussion & $\begin{array}{c}0.06 * * * \\
(0.02)\end{array}$ & $\begin{array}{c}0.02 \\
(0.02)\end{array}$ & $\begin{array}{c}0.05^{* * *} \\
(0.02)\end{array}$ & $\begin{array}{c}0.02 \\
(0.02)\end{array}$ \\
\hline Income & $\begin{array}{c}0.00 \\
(0.00)\end{array}$ & $\begin{array}{l}-0.01 * \\
(0.00)\end{array}$ & $\begin{array}{c}0.00 \\
(0.00)\end{array}$ & $\begin{array}{c}-0.01 * * \\
(0.00)\end{array}$ \\
\hline Education & $\begin{array}{c}-0.01 * * \\
(0.00)\end{array}$ & $\begin{array}{c}0.01 * * * \\
(0.00)\end{array}$ & $\begin{array}{c}-0.01 * * \\
(0.00)\end{array}$ & $\begin{array}{c}0.01 * * \\
(0.00)\end{array}$ \\
\hline Age & $\begin{array}{c}0.00^{* * *} \\
(0.00)\end{array}$ & $\begin{array}{c}0.00 * * * \\
(0.00)\end{array}$ & $\begin{array}{c}0.00^{* * *} \\
(0.00)\end{array}$ & $\begin{array}{c}0.00^{* * *} \\
(0.00)\end{array}$ \\
\hline Religiosity & $\begin{array}{c}0.02 * * * \\
(0.00)\end{array}$ & $\begin{array}{c}0.00 \\
(0.00)\end{array}$ & $\begin{array}{c}0.02 * * * \\
(0.00)\end{array}$ & $\begin{array}{c}0.00 \\
(0.00)\end{array}$ \\
\hline Urban Density & $\begin{array}{c}0.00 \\
(0.00)\end{array}$ & $\begin{array}{c}-0.01 * * * \\
(0.00)\end{array}$ & $\begin{array}{c}0.00 \\
(0.00)\end{array}$ & $\begin{array}{c}-0.01 * * * \\
(0.00)\end{array}$ \\
\hline French & $\begin{array}{c}-0.08 * * * \\
(0.03)\end{array}$ & $\begin{array}{c}-0.09 * * * \\
(0.03)\end{array}$ & $\begin{array}{c}-0.09 * * * \\
(0.02)\end{array}$ & $\begin{array}{c}-0.08 * * * \\
(0.03)\end{array}$ \\
\hline Female & $\begin{array}{c}0.00 \\
(0.01)\end{array}$ & $\begin{array}{c}0.09 * * * \\
(0.01)\end{array}$ & $\begin{array}{l}-0.01 \\
(0.01)\end{array}$ & $\begin{array}{c}0.09 * * * \\
(0.01)\end{array}$ \\
\hline Pseudoscience & & & $\begin{array}{c}0.07 * * * \\
(0.02)\end{array}$ & $\begin{array}{l}-0.01 \\
(0.03)\end{array}$ \\
\hline Anti-intellectualism & & & $(0.03)$ & $\begin{array}{c}-0.09 * * * \\
(0.03)\end{array}$ \\
\hline Science Literacy & & & $\begin{array}{c}-0.05^{* * *} \\
(0.02)\end{array}$ & $\begin{array}{c}0.01 \\
(0.02)\end{array}$ \\
\hline Ideology & & & $\begin{array}{c}-0.14 * * * \\
(0.02)\end{array}$ & $\begin{array}{l}-0.00 \\
(0.02)\end{array}$ \\
\hline Constant & $0.77 * * *$ & $0.63^{* * *}$ & $0.88^{* * *}$ & $0.69 * * *$ \\
\hline $\mathrm{R}^{2}$ & 0.11 & 0.29 & 0.16 & 0.30 \\
\hline $\mathrm{N}$ & 2022 & 2022 & 2022 & 2022 \\
\hline
\end{tabular}

